\title{
Adulthood Presentation of Bilateral Complete Congenital Choanal Atresia with other Congenital Anomalies: A Case Report with Review of Literature
}

\author{
${ }^{1}$ Vinish Kumar Agarwal, ${ }^{2}$ Sampan Singh Bist, ${ }^{3}$ Sarita Kumari Mishra, ${ }^{4}$ Bhartendu Bharti
}

\begin{abstract}
Bilateral choanal atresia is an otorhinolaryngology emergency. It is a potentially life-threatening disorder because the affected newborn is an obligate nose breather. We report clinical and radiological evaluation of a patient with congenital bilateral choanal atresia, who presented at the 20-year of age with other congenital anomalies. Patient was diagnosed as congenital bilateral choanal atresia, right accessory nasal deformity, high arched palate with congenital phthisis bulbi of his left eye. He was posted for transnasal endoscopic surgery under general anesthesia. Unique feature of this case is left congenital phthisis bulbi along with bilateral congenital choanal atresia probably the first case report having both anomalies in a single patient. While performing surgery, we should carefully study the anatomy of the basisphenoid, as in our case we did not attempt to correct atresia on right side.
\end{abstract}

Keywords: Congenital choanal atresia, Congenital phthisis bulbi, Transnasal endoscopic sinus surgery.

How to cite this article: Agarwal VK, Bist SS, Mishra SK, Bharti B. Adulthood Presentation of Bilateral Complete Congenital Choanal Atresia with other Congenital Anomalies: A Case Report with Review of Literature. Clin Rhinol An Int J 2015;8(1):24-26.

\section{Source of support: Nil \\ Conflict of interest: None}

\section{INTRODUCTION}

Bilateral congenital choanal atresia is considered a lethal congenital malformation as newborns are generally obligate nasal breathers for the first few months of life so, it will present as an acute respiratory emergency at birth with classical picture of cyclical cyanosis (blue spells relieved by crying). We present a case of 20-year aged male with bilateral congenital choanal atresia with congenital right accessory nasal deformity, high arched palate and left congenital phthisis bulbi.

\footnotetext{
${ }^{1,3,4}$ Assistant Professor, ${ }^{2}$ Professor and Head

${ }^{1-4}$ Department of ENT, Himalayan Institute of Medical Sciences Dehradun, Uttarakhand, India
}

Corresponding Author: Vinish Kumar Agarwal, Assistant Professor, Department of ENT, Himalayan Institute of Medical Sciences, Dehradun, Uttarakhand, India, Phone: 01352471371, e-mail: vinish143agra@yahoo.co.in

\section{CASE REPORT}

A 20-year aged male presented with nasal deformity, mouth breathing and snoring (Fig. 1). This patient, second child of three siblings, was born at home as full-term normal vaginal delivery with uneventful antenatal, perinatal and postnatal period. He cried immediately after birth and had no difficulty in feeding. Parents went to primary healthcare center just after the birth of the child for external deformity of nose and eye. This child was referred to higher center but parent never turned up until now. There is no significant family history.

On examination, there was wide dorsum of nose with no airflow in both of his anterior naris and severe rhinolalia clausa. There were three nostrils, right being divided in two by a barrier diaphragm arising from inferior turbinate (Fig. 2). The medial part of right naris was connected to nasal cavity while lateral part did not communicate. This patient also had very high arched palate and left small blind eye which was diagnosed as congenital phthisis bulbi.

On diagnostic nasal endoscopy posterior choanae could not be identified, and drops instilled in nasal cavity could not be perceived by patient in throat and suction catheter could not be passed in nasopharynx through either of nasal cavity (Fig. 3). So, clinical diagnosis of bilateral choanal atresia was made in this case. Computed tomography (CT) scan of nose and paranasal sinus was done which showed complete bilateral bony atretic plate at the posterior end of both side nasal cavities (Fig. 4).

So, this patient was diagnosed as congenital bilateral choanal atresia, right accessory nasal deformity, high arched palate with congenital phthisis bulbi of his left eye. He was posted for transnasal endoscopic surgery under general anesthesia.

During the surgery, barrier diaphragm was removed on right side and the right anterior naris was recovered. A bony hard atretic plate was found to block both posterior nares. Vomer bone at the posterior end of septum was removed. Bony atretic plate on right side was in direct contact with basisphenoid, as there was no nasopharynx on right side according to CT scan, so, the atretic plate was perforated with microdebrider and diamond paste burr on left side only. Left nasal 


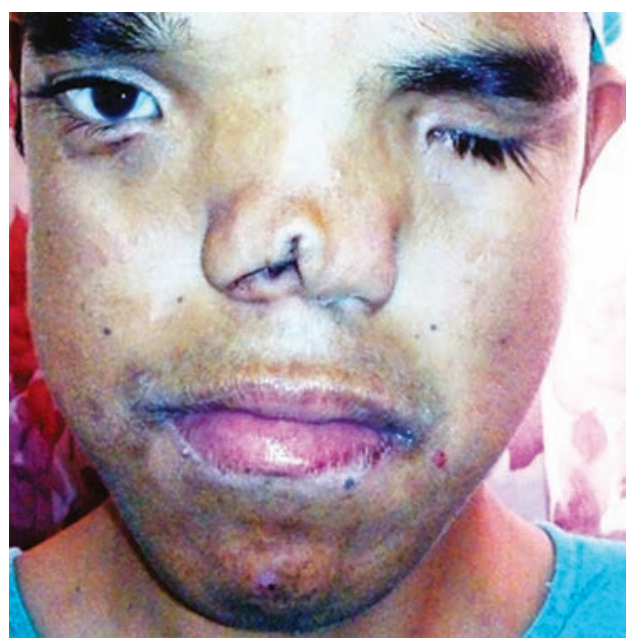

Fig. 1: Clinical profile of patient

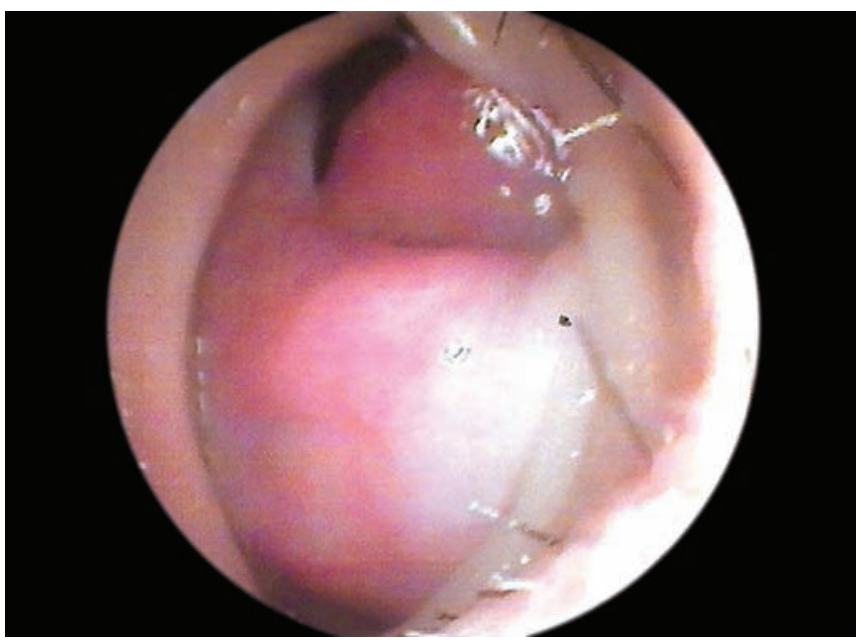

Fig. 3: Bony atretic plate at choanae

stent was fashioned from portex endotracheal tube cut to length. The stent were secured by a circumseptal ' 0 ' prolene suture and were left in situ for 6 weeks. After 6 weeks, portex tube was removed and on nasal endoscopy well epithealized posterior choanae were noted on left side. Rhinolalia clausa and sleep snoring was all markedly relieved. Patient is on regular monthly follow-up presently (Fig. 5).

\section{DISCUSSION}

The etiology of congenital atresia is unknown. Three embryologic theories have been advanced the most logical being that a medial overgrowth of the vertical and horizontal process of the palatal bones results in choanal atresia. This theory, however, fails to explain membranous choanal atresia. It is of interest that associated congenital anomalies occur with congenital choanal atresia in $43 \%$ of the case reports. ${ }^{1}$ Overall, incidence of choanal atresia being 1 in 7000 live births, 90\% of atresia are bony while $10 \%$ being membranous. ${ }^{2}$

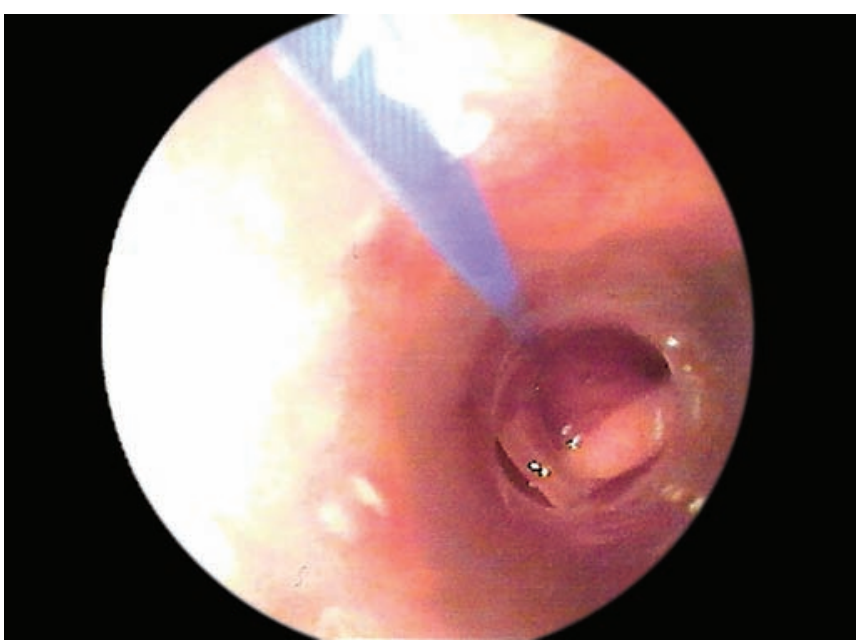

Fig. 2: Bifid nasal septal deformity

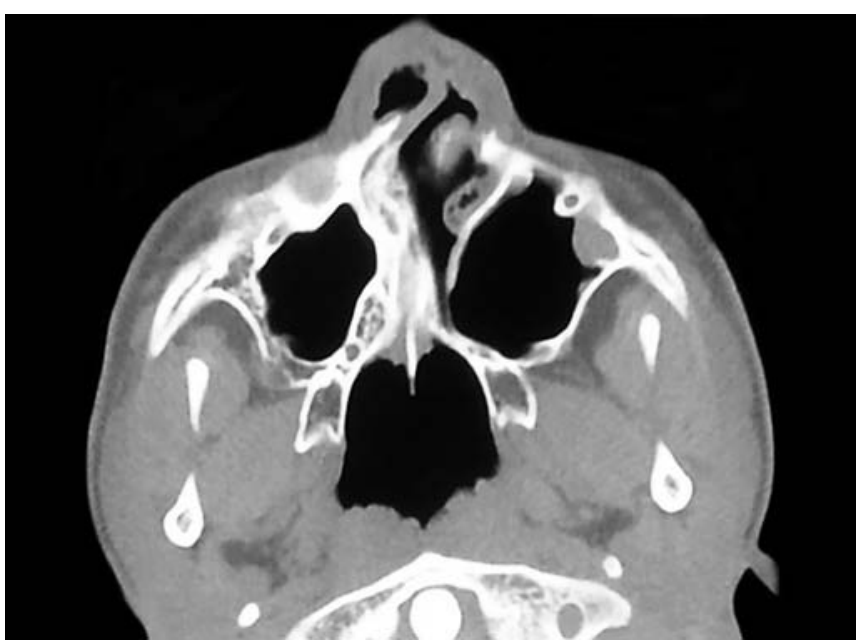

Fig. 4: Axial section showing choanal atresia

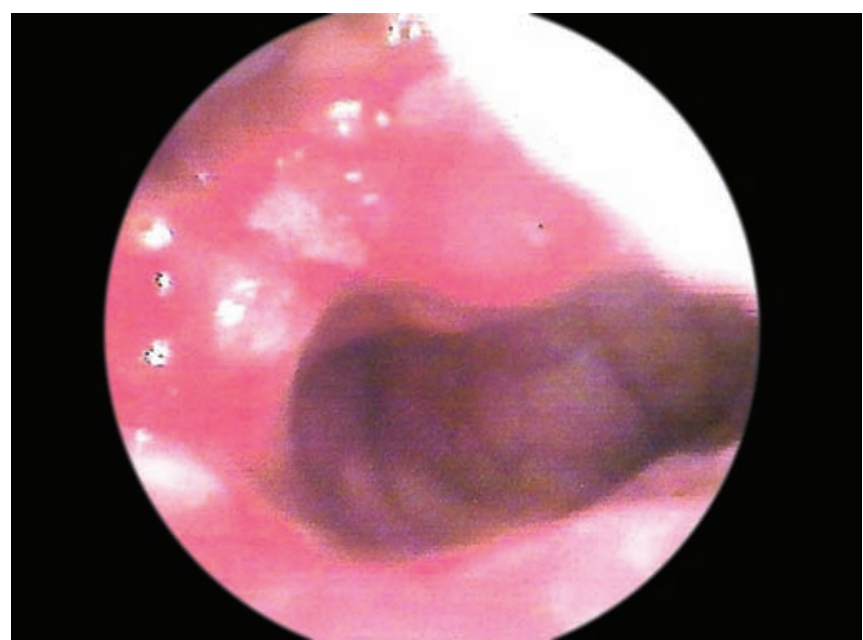

Fig. 5: Twelve weeks postoperative image

Bilateral choanal atresia is an otolaryngology emergency. It is a potentially life-threatening disorder because the affected infant is an obligate nose breather. ${ }^{3}$ Symptoms range from intermittent to severe respiratory distress with cyanosis that is aggravated by feeding and alleviated by crying. Approximately, $75 \%$ of children with 
bilateral choanal atresia have other congenital abnormalities, as exemplified by Charge syndrome (coloboma of eye or microphthalmia, heartmalformation, choanal atresia, retarded growth, genital hypoplasia, and ear abnormalities, typically external). ${ }^{4}$ In our case, patient did not had respiratory difficulty after the birth because of other congenital anomaly of palate provided the compensatory mechanism facilitating the oropharyngeal respiration. Surgical correction of bilateral choanal atresia is performed as soon as possible after the diagnosis has been made; however, there is little consensus regarding the optimal surgical approach. ${ }^{5}$ Transpalatal, transnasal, transseptal, and sublabial approaches have been utilized with varying degrees of success. ${ }^{5}$ Recently, the transnasal endoscopic procedure has been advocated as a safe and efficacious method with the best possibility for long-term nasal patency.,6

Congenitalaccessorynasaldeformity isasupernumerary malformation in the external nose. Supernumerary malformation may appear in different ways. It may replace the normal structure or co-exist with it. Removal of the congenital accessory is the optimal treatment method. ${ }^{7}$ The patient in our report bore three anterior nares, however, this should be differentiated from three-nostril malformation, a type of cleft nose occurring when the median line of the nasal ridge presents as a major groove. In our case, only the soft tissue formed the malformation in one side of the anterior nares, therefore, this case was diagnosed as 'congenital accessory nasal deformity'.

\section{Congenital Phthisis Bulbi}

Phthisis bulbi as a result of some suppurative traumatic or non-traumatic uveal inflammation is not a very rare condition, but shrinking of the globe as a result of uveal inflammation in utero is very rare indeed, and deserves to be recorded in medical literature. ${ }^{8}$ Congenital microphthalmia should be distinguished from phthisis bulbi, which usually represents an acquired shrinkage of the eye following trauma, inflammation, and so forth rather than a primary development defect. The standardized A-scan and B-scan echography help us to distinguish the phthisis bulbi from atrophy of the eyeball without shrinkage and atrophy of the eyeball with shrinkage.

\section{CONCLUSION}

Congenital bilateral choanal atresia is a life-threatening disease in newborns. However, it can also be found in adults, who may present with mouth breathing, sleep snoring and symptoms of nasal obstruction. Choanal atresia is often accompanied by other congenital abnormalities. In our case, we have a 20 -year aged patient with congenital bilateral choanal atresia with right supernumerary accessory nostril with right external nose deformity along with high arch palate. But unique feature of our case is left congenital phthisis bulbi along with bilateral congenital choanal atresia probably the first case report having both anomalies in a single patient. While performing surgery, we should carefully study the anatomy of the basisphenoid, as in our case we did not attempt to correct atresia on right side.

\section{REFERENCES}

1. Blasberg B, Stool S, Oka S. Choanal atresia, a cryptic congenital anomaly. Cleft Palate J 1975;12:409-416.

2. Vanzieleghem BD, Lemmerling MM, Vermeersch HF, Govaert P, Dhooge I, Meire F, Mortier GR, Leroy J, Kunnen MF. Imaging studies in the diagnostic workup of neonatal nasal obstruction. J Comput Assist Tomogr 2001;25:540-549.

3. Neskey D, Eloy JA, Casiano RR. Nasal, septal, and turbinate anatomy and embryology. Otolaryngologic Clinics of North America, 2009;42(2)193-205.

4. Burrow TA, Saal HM, De Alarcon A, Martin TJ, Cotton RT, Hopkin RJ. Characterization of congenital anomalies in individuals with choanal atresia. Archives of Otolaryngol 2009;135(6):543-547.

5. Ramsden JD, Campisi P, Forte V. Choanal atresia and choanal stenosis. Otolaryngologic Clinics of North America 2009; 42(2):339-352.

6. Friedman NR, Mitchell RB, Bailey CM, Albert DM, Leighton DEJ. Management and outcome of choanal atresia correction. Int J Pediat Otorhinolaryngol 2000;52(1):45-51.

7. Tambwekar SR, Aiyer PM, Vij VK. Supernumerary nostril in association with incomplete naso-ocular cleft. Plast Reconstr Surg 1997;100:682-684.

8. Brav A. Congenital phthisis bulbi with report of a case. JAMA 1914;63(22):1949-1950. 